\title{
Flexible Test Bed for MVDC and HFAC Electric Ship Power System Architectures for Navy Ships
}

\author{
John D. Herbst, Angelo L. Gattozzi, A. Ouroua, Fabian M. Uriarte \\ The University of Texas at Austin \\ Center for Electromechanics \\ Austin, Texas USA \\ j.herbst@cem.utexas.edu
}

\begin{abstract}
Several power architectures have been considered for Navy ships and significant effort has been focused on simulation of the various power system topologies. The University of Texas at Austin Center for Electromechanics (UT-CEM) has taken a step forward by assembling a microgrid capable of operating at MW power levels to experimentally validate key elements of these system models. The present system is an MVDC architecture but can easily be reconfigured as an HFAC network. This paper describes the UT-CEM microgrid and plans to demonstrate critical technical issues in naval power systems and enable model validation. The intent is for the microgrid to be a flexible test bed for investigation of naval power systems and to become a useful bridge from theoretical and computer studies to a realistic experimental platform.
\end{abstract}

\section{INTRODUCTION}

As part of the Electric Ship Research and Development Consortium (ESRDC), the University of Texas at Austin Center for Electromechanics (UT-CEM) has been conducting high fidelity simulations of naval electric power system architectures. This work builds on a long history of power system modeling and the development and demonstration of advanced power generation and conversion technologies and systems. The ESRDC is currently evaluating system trades between medium voltage dc (MVDC) and high frequency ac (HFAC) power systems relative to conventional $60 \mathrm{~Hz}$ electric power distribution systems. These isolated grid systems typically operate at power levels of 80-100 MW with a variety of steady state and pulsed power loads. The simulation models allow exploration of novel system architectures and hardware that may not be available at the required power levels - or in some cases may not yet exist at all. As with any computer simulation of large real world systems, it is important to anchor the simulations with experimental data to validate the simulation results and provide confidence that the simulations can be extended to operating regimes beyond the experimental demonstrations.

Technical risk, time and cost constraints typically prevent validation of system simulations through tests with full scale system hardware, so subscale experimental systems are used for the validation experiments. While low power tests can provide insight into system performance, experiments conducted at higher power levels can often reveal behavior that is not evident at the power levels seen in bench top experiments. To support the ESRDC modeling efforts, UTCEM has now taken a further step forward by assembling a microgrid demonstrator capable of operating at MW power levels in order to experimentally validate key elements of these system models and component studies. The microgrid is designed as a flexible system, easily expandable in power and re-definable in terms of voltage levels and system configuration. This paper presents a description of the UTCEM microgrid and planned experiments for model validation.

\section{MOTIVATION}

UT-CEM has been an active contributor to the generation and analysis of naval power system studies for many years. This work has been based on the conviction that modeling and simulation play a critical role in understanding complex shipboard electrical power structures. Development of a microgrid test bed is another decisive step forward in the development of reliable shipboard power systems. In fact, due to the obvious complexities and the variety of system architectures under study, it is fair to say that while the theoretical understanding of the issues involved and the computer simulation efforts have been pursued actively by the technical community at large, the experimental database needed to validate the analytical models and support the large scale implementation of such assemblies is still very limited. The UT-CEM microgrid can provide a bridge between the theoretical study of these systems and subsequent phases of the technology development process at MW power levels.

\section{MICROGRID DESCRIPTION}

The major elements of the microgrid (Fig. 1) were assembled using existing components available from prior research activities at UT-CEM. These components are 
typically identified by the original project name. For example, "ALPS passive rectifier" refers to a diode rectifier developed for the Advanced Locomotive Propulsion System (ALPS)

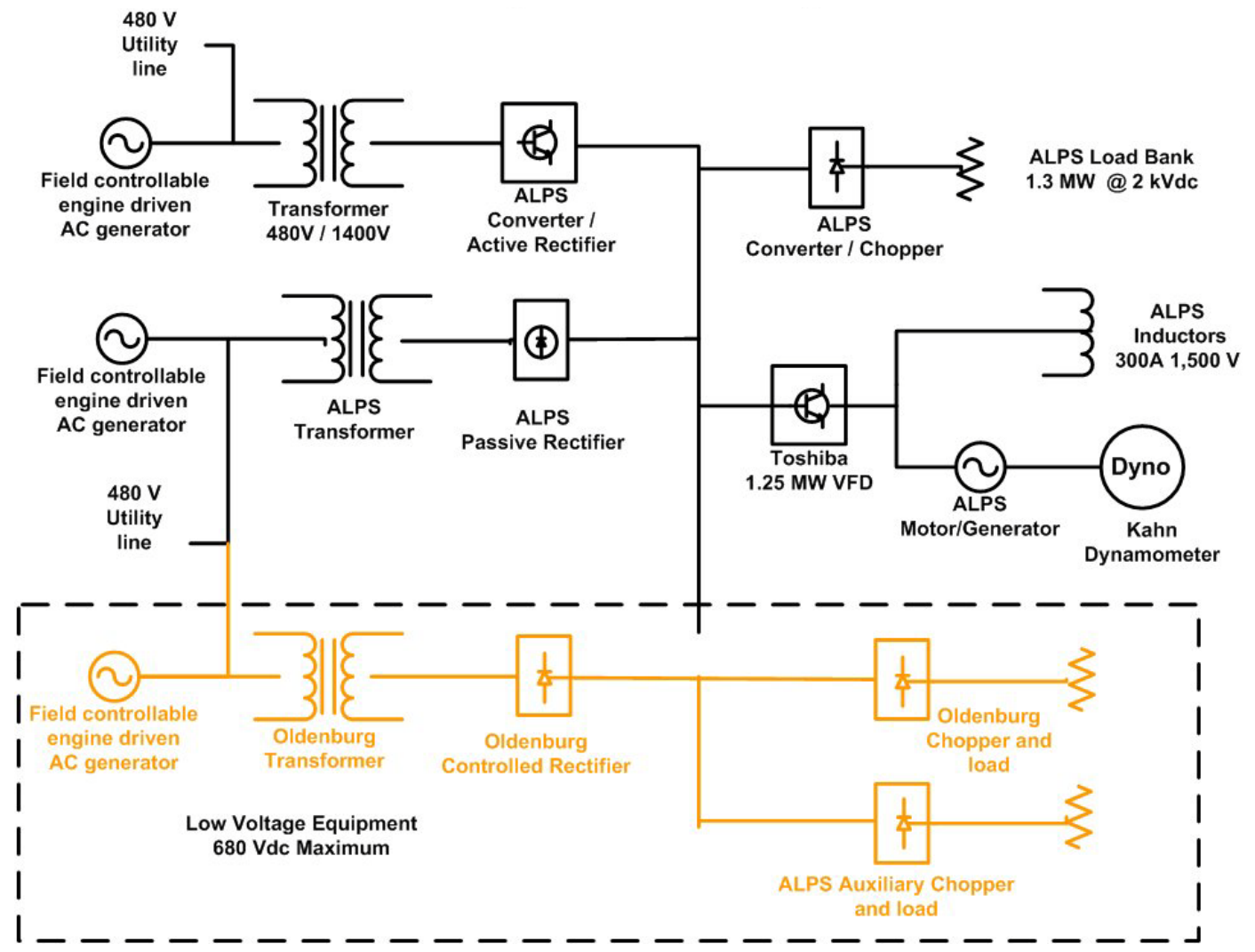

Figure 1. UT-CEM microgrid demonstrator circuit schematic.

The microgrid is currently configured around a central $2 \mathrm{kV}$ dc bus that incorporates multiple power sources and a variety of active and passive loads. The microgrid distribution bus spans over $150 \mathrm{ft}$. between two separate labs within the UT-CEM facility to provide spatial separation of the power system elements and realistic bus inductance/capacitance values. Key elements of the microgrid are described in the following sections.

\section{A. Power Sources}

The microgrid is currently configured with two independent $480 \mathrm{~V}, 60 \mathrm{~Hz}$, three-phase utility power supplies connected through transformers with multiple secondary windings. The power supply interfaces are located at either end of the distribution bus: one in the main microgrid lab and the second in the gas turbine/high speed generator test cell. Appropriate configuration of the input and output windings enables a broad range of secondary ac voltages. The range of possible ac secondary voltages and the corresponding dc voltages are shown in Table 1.

In addition to the $480 \mathrm{~V}$ utility power supply, the system has been configured to allow the use of diesel-electric generators to provide power to the transformer primary input windings. The use of diesel-electric generators will allow demonstration of power systems where the power source is of comparable scale to the installed loads. This is typical of shipboard power systems where the installed power is often program. Some of these components are rated for operation only up to $480 \mathrm{~V}$; these items are shown in the dashed box of Fig. 1. less than the total of the electric loads of the ship power system.

TABLE I. MiCROGRID DISTRIBUTION VOLTAGES

\begin{tabular}{|c|c|}
\hline $\begin{array}{c}\text { AC Secondary } \\
\text { [Vac] }\end{array}$ & $\begin{array}{c}\text { Dc Volts } \\
\text { [Vdc Peak] }\end{array}$ \\
\hline 404 & 571 \\
\hline 480 & 679 \\
\hline 700 & 990 \\
\hline 808 & 1143 \\
\hline 1,212 & 1714 \\
\hline 1,400 & 1980 \\
\hline 1,616 & 2285 \\
\hline 2,100 & 2970 \\
\hline 2,435 & 3444 \\
\hline 2,800 & 3960 \\
\hline 3,637 & 5143 \\
\hline 4,850 & 6859 \\
\hline
\end{tabular}

In addition to these two relatively conventional power sources, the UT-CEM microgrid can also be configured for high frequency ac (HFAC) power distribution using high speed, high frequency generators directly driven from gas turbine prime movers. UT-CEM has been actively developing high speed rotating electric machines for a variety of applications for over 35 years. Fig. 2 is a picture of a gasturbine-driven $3 \mathrm{MW}$ high speed generator during testing on the ALPS program. 


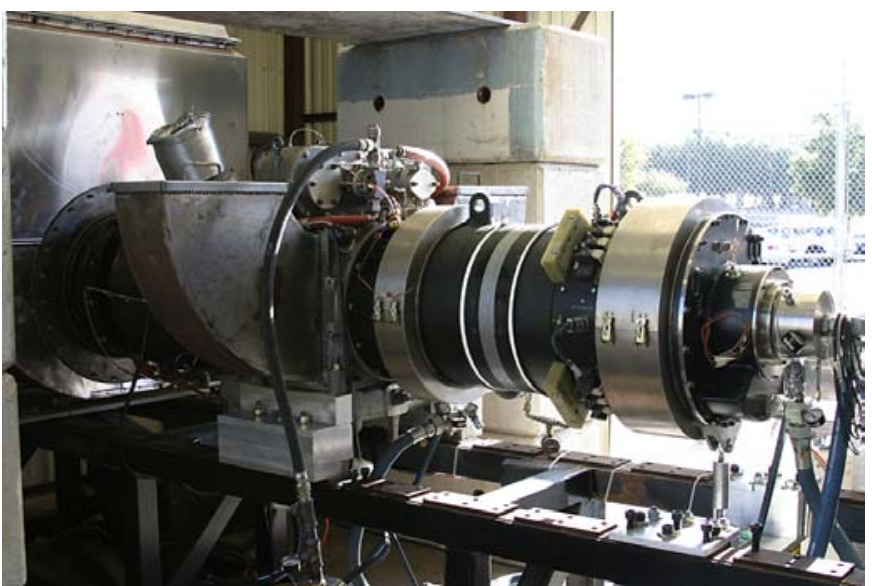

Figure 2. ALPS $3 \mathrm{MW}$ high speed generator testing at UT-CEM.

\section{B. Power Conversion Modules}

The microgrid features two independent power conversion modules: a passive full bridge diode rectifier and an active IGBT controlled rectifier. (An additional controlled rectifier is available at lower voltages.) The power conversion modules were originally developed as elements of a hybrid-electric propulsion system for a high speed passenger locomotive under the Advanced Locomotive Propulsion System (ALPS) program. Fig. 3 shows the passive rectifier and bidirectional power converter adapted for use with the microgrid.

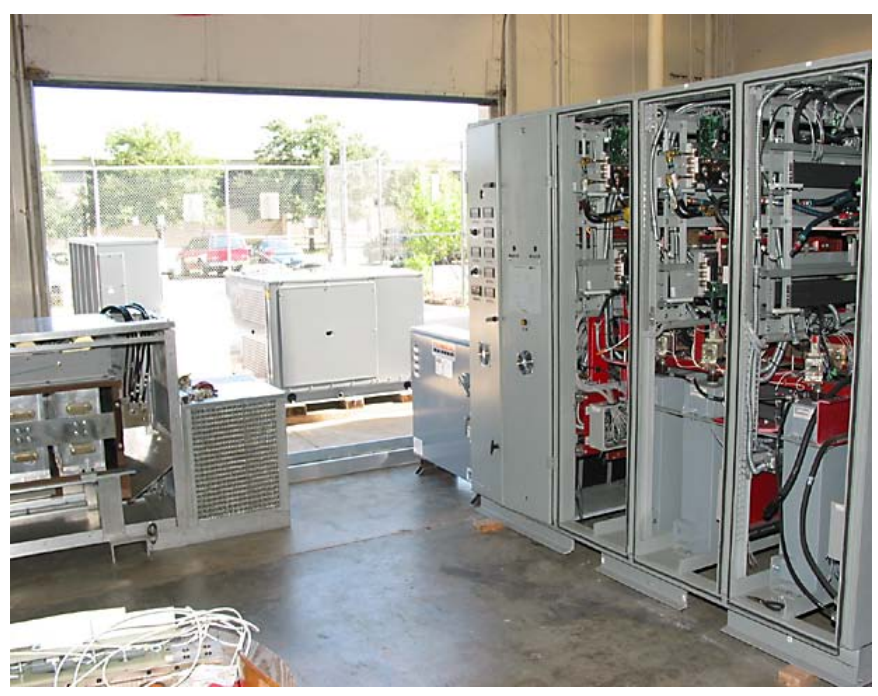

Figure 3. Passive rectifier (left) and bi-directional power converter.

The microgrid also includes a Toshiba Model G7 Variable Frequency Drive (VFD) rated at $1.25 \mathrm{MW}$ and capable of output frequencies up to $300 \mathrm{~Hz}$. The commercial VFD has been modified to allow for direct interface to the internal dc bus to allow the drive to be powered directly from the microgrid dc bus. The VFD enables evaluation of solid state switched loads on the distribution bus. The VFD drives a high speed induction machine rated at $2 \mathrm{MW}$ at speeds ranging from
7,500 to $15,000 \mathrm{rpm}$. The induction machine originally served as the motor/generator for a large energy storage flywheel developed for the ALPS program.

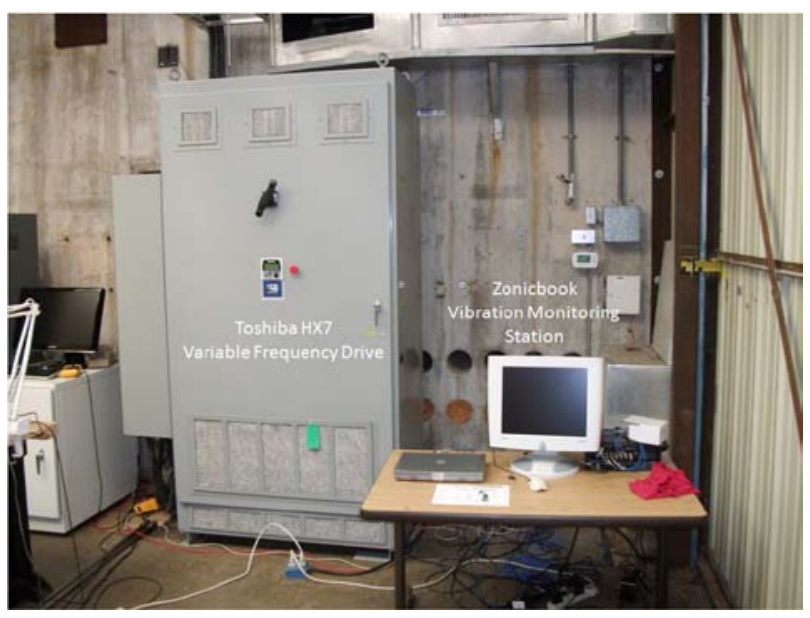

Figure 4. Toshiba Model G7 Variable Frequency Drive.

\section{Loads}

The microgrid features a variety of resistive, inductive and switched dynamic loads. Resistive loads can be configured as static fixed loads on the bus or they can be modulated through controlled choppers. The primary resistive load is an air-cooled locomotive dynamic brake resistor rated to dissipate $1.3 \mathrm{MW}$ at 2,000 Vdc. The brake resistor load is controlled by a chopper circuit located in the main power converter. An array of form wound coil inductors can provide inductive loads of

- $3.2 \mathrm{mH}$ per phase Y-connected at $600 \mathrm{~A}$

- $6.4 \mathrm{mH}$ per phase Y-connected at $300 \mathrm{~A}$

- $12.8 \mathrm{mH}$ per phase Y-connected at $300 \mathrm{~A}$

Dynamic switched loads for the microgrid are provided by a high speed induction motor and Kahn Model 108-080 hydraulic dynamometer. The dynamometer is rated to absorb up to $4.77 \mathrm{MW}$ at speeds up to $18,000 \mathrm{rpm}$. The dynamometer is currently configured for vertical orientation to match the high speed induction machine, but can also be mounted in a horizontal orientation to mate with conventional horizontalaxis motors. Fig. 5 is a picture of the induction motor and dynamometer in the UT-CEM spin test bunker. The dynamometer controller enables programmable dynamic load profiles to create transient loads on the microgrid. 


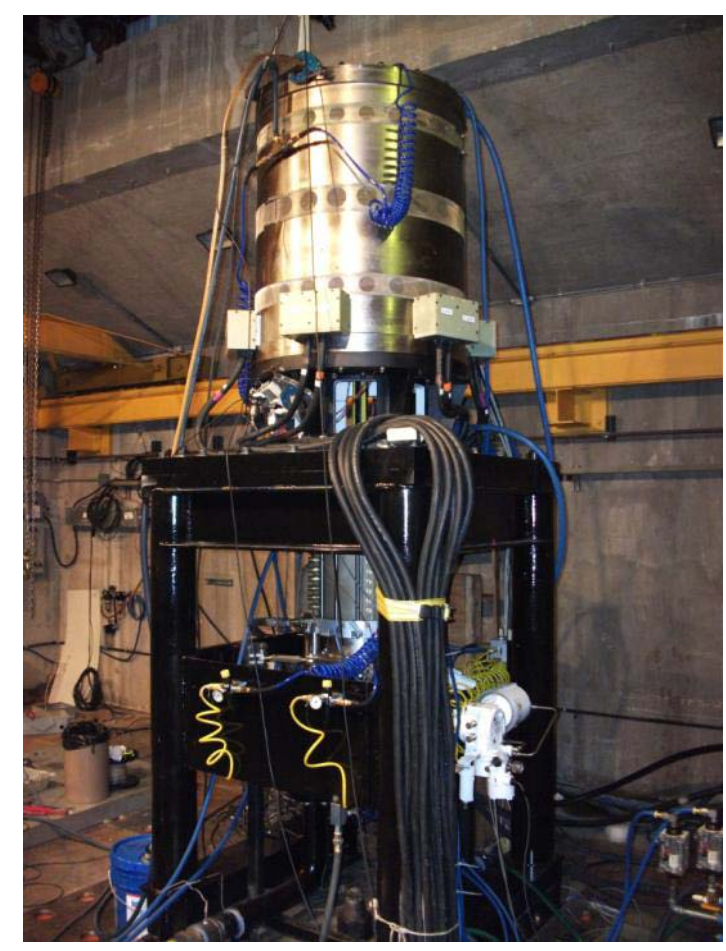

Figure 5. High speed induction motor and hydraulic dynamometer.

\section{Instrumentation and Control}

The instrumentation and control system for the microgrid is based on National Instruments' LabView platform. LabView provides a flexible control system that can easily be adapted as the configuration of the microgrid is changed. Monitoring of critical system parameters is supported by a suite of data acquisition and instrumentation equipment specifically selected for use with advanced power systems. Procurement of the Energy Storage Instrumentation System (ESIS) was funded under a Defense University Research Instrumentation Program (DURIP) grant sponsored by ONR. Table 2 is a partial list of the ESIS equipment.

\section{TABLE II. ESIS PARTIAL EQUIPMENT LIST}

\begin{tabular}{|c|c|}
\hline DESCRIPTION & Qty. \\
\hline Bently Nevada 3300 XL Proximitor Probes & 8 \\
\hline PCB Piezotronics Model 356A25 accelerometers & 4 \\
\hline PCB Piezotronics Model 338C04 accelerometers & 8 \\
\hline ZONICBOOK/618 EZT 8-channel system & 2 \\
\hline Monarch Instrument tachometers SPS-5/115 & 2 \\
\hline Mixed Signal Oscilloscope, Agilent MSO7054A & 1 \\
\hline Portable oscilloscope, Fluke 199C003S & 1 \\
\hline North Star PVM-11 high voltage probes & 16 \\
\hline Fluke i300flex current probes & 4 \\
\hline Voltech Instruments PM6000 Power Analyzer & 1 \\
\hline LEM transducers, LT 1005-S & 6 \\
\hline FLIR ES320 thermal imaging system & 1 \\
\hline Digital Storage oscilloscope, Agilent DSO6054L & 1 \\
\hline CompactRio Control and Acquisition System & 1 \\
\hline
\end{tabular}

\section{EXPERIMENTAL PROGRAM}

\section{A. Experimental Activities}

The microgrid is currently configured to support evaluation of series faults in both ac and dc distribution systems. Series faults - such as a failing bus or cable connection - can be difficult to detect until the associated arcs have caused significant damage. UT-CEM developed a test fixture (Fig. 6) that enables controlled separation of copper electrodes to simulate a series fault in the distribution bus. Fig. 7 is a picture taken during a series fault experiment.

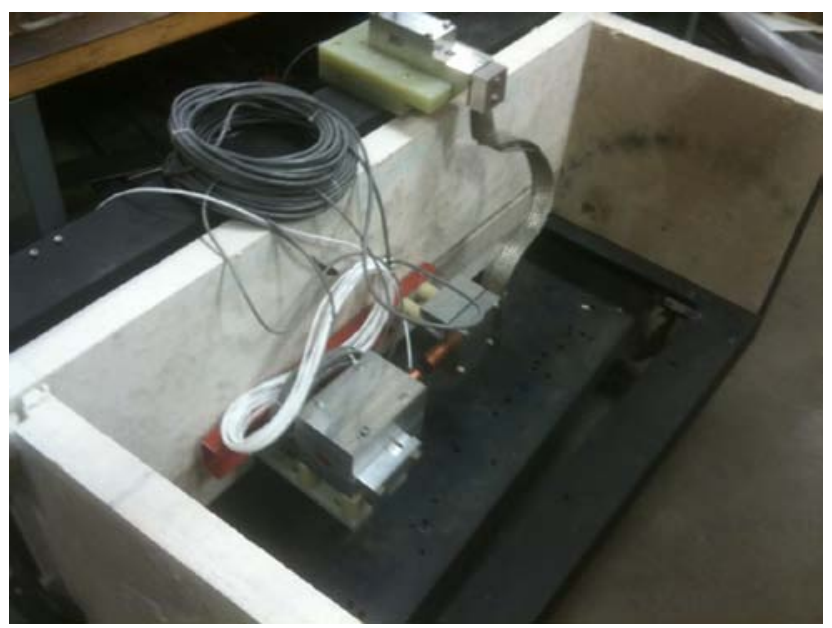

Figure 6. Series fault experimental fixture.

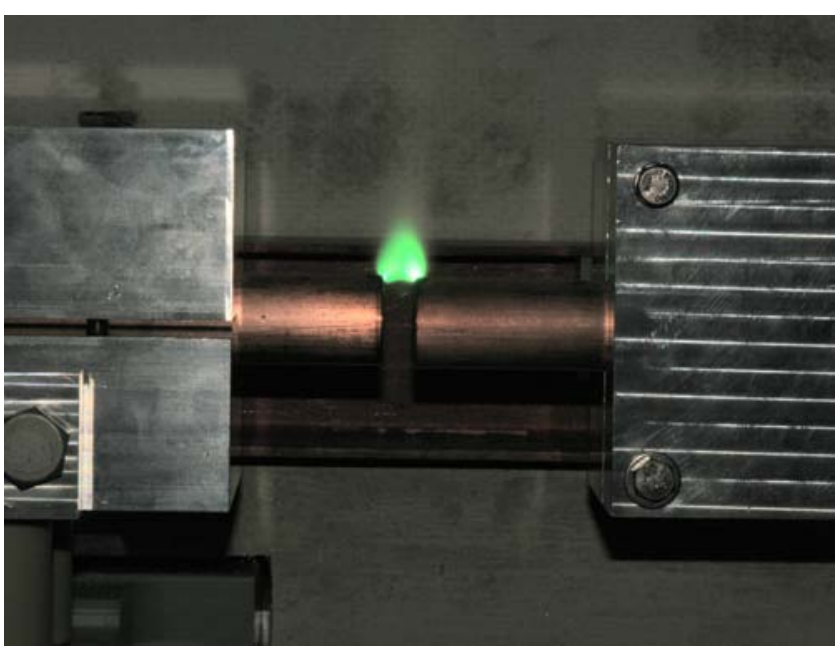

Figure 7. Series fault experiment showing arc between electrodes.

MatLab Simulink models of the microgrid circuit and series fault have been developed and experiments are currently being conducted to validate and refine the computer model of the arc fault in both ac and dc distribution systems. Simulation efforts include two types of faults. One is a longitudinal tear with constant speed to a predetermined fixed spacing. This simulates a power system that is well supported mechanically, 
but initiates an arc due to failure of a conductor or connector because of circumstances such as excessive heat or corrosion. This case is expected to cause few electrical problems, but can be a source of fire as shown in Fig. 8.

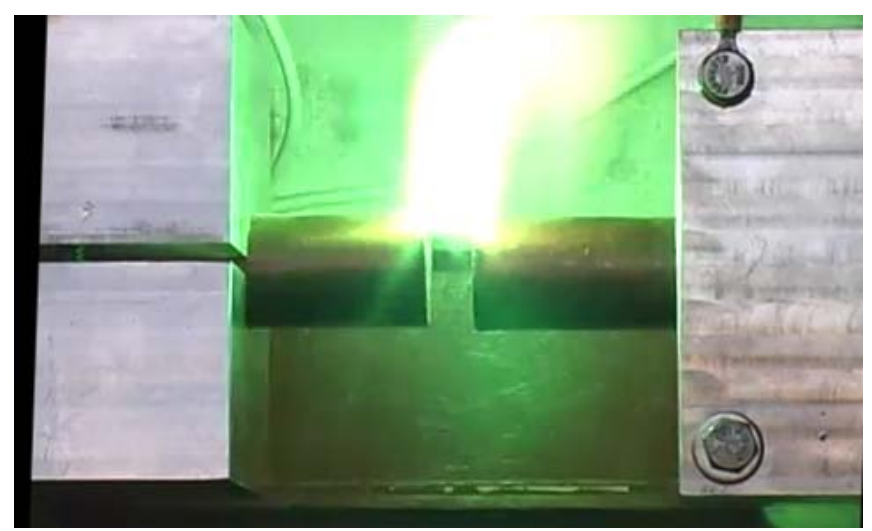

Figure 8. Longitudinal conductor tear at 0.1 in./s separation velocity.

An initial experiment was carried out at a grid bus voltage of $280 \mathrm{Vdc}$ with an equivalent load impedance of $Z \approx 8+j 0.2 \Omega$, a resistance of $8 \Omega$ in series with a $600 \mu \mathrm{H}$ inductance. Before energizing the microgrid, several simulations were run to predict the influence of the gap's voltage and current envelopes. These initial simulations are based on prior experimental work with the series fault test fixture under different conditions. An illustration of the simulation results is shown in Fig. 9. Experimental voltage and current waveforms are shown in Fig. 10. Discrepancies between simulated and measured results exist because the fault model is based on empirical data from prior tests which have been forward-ported to predict fault behavior in the present context. The early test circuit did not include the inductance and capacitance associated with the extended dc bus and larger load resistor. The early experiments provided the basis for the initial simulation models and we are developing updated models to reflect the latest experimental data taken from the microgrid circuit at relatively low voltages. The updated models will then be used to predict current and voltage waveforms in higher power experiments on the microgrid.

With the same grid voltage and load, the second case was that of a longitudinal fault with separation acceleration of approximately $9.8 \mathrm{~m} / \mathrm{s}^{2}$. This reflects a situation such as an energized cable that breaks and falls as shown in Fig. 11. The arc will extinguish, but it is important to know whether the rate of change of voltage or current is sufficient to cause harmful transients in the power system. Preliminary experimental results from the accelerating arc gap are shown in Fig. 12.

We are currently verifying the performance of the test fixture and data acquisition systems under these conditions and developing models to enable predictions of voltage and current waveforms for this case. An initial observation from the testing is that these fault types do not appear to produce harmful $\mathrm{dv} / \mathrm{d} t$ and $\mathrm{di} / \mathrm{d} t$ transients with the system parameters tested.

These tests will be repeated at progressively higher grid voltages, for both cases of a dc and HFAC bus, in order to thoroughly assess the impact that a series fault may have on the system.

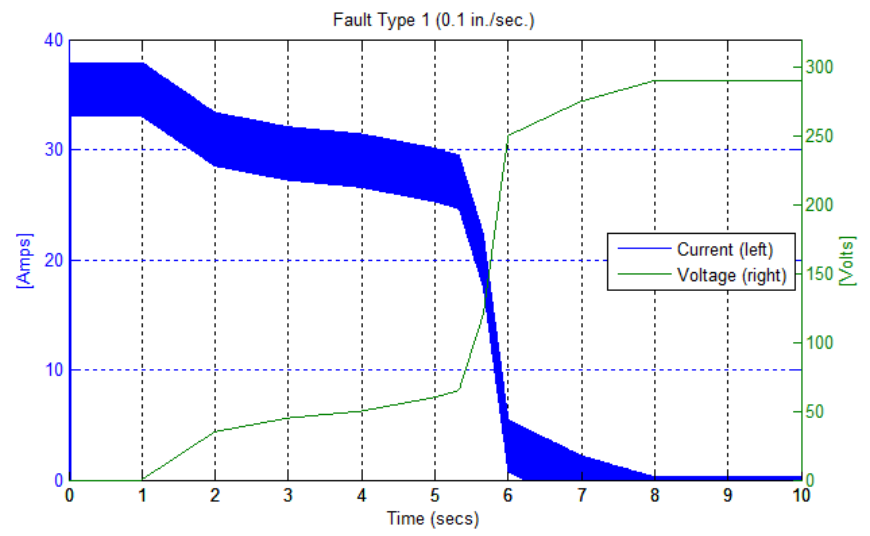

Figure 9. Simulated gap voltage and current for fault in Fig. 8.

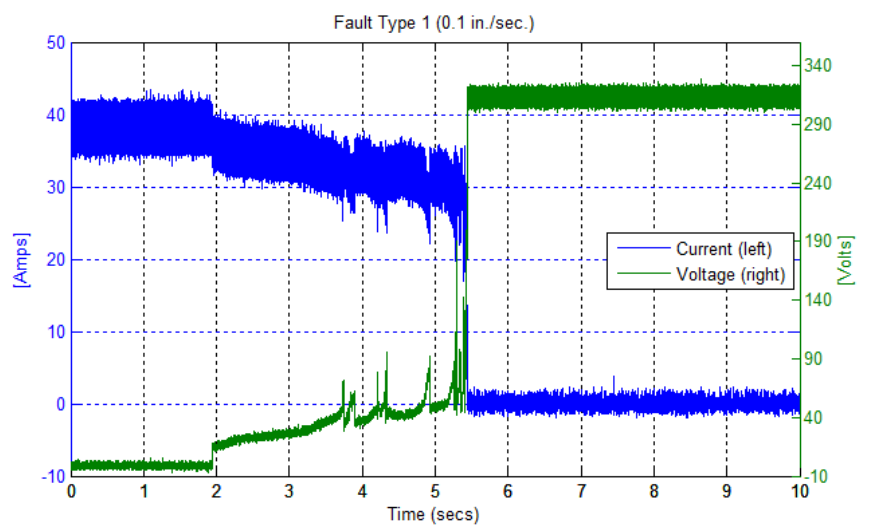

Figure 10. Measured gap voltage and current for fault in Fig. 8.

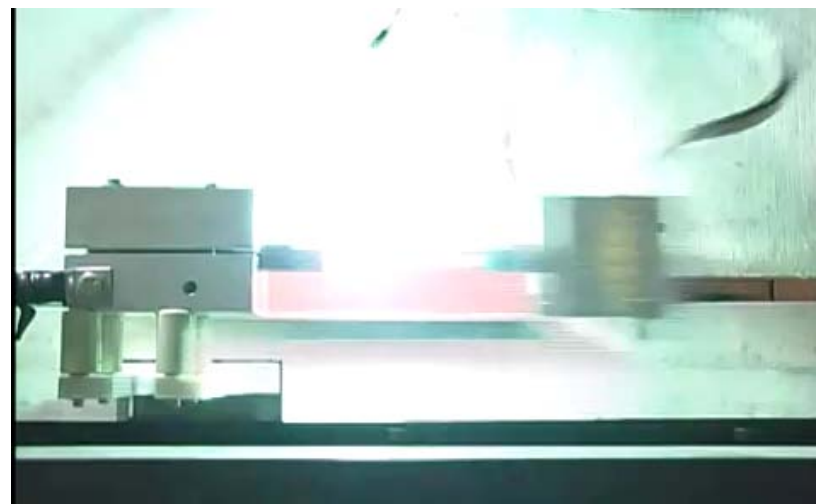

Figure 11. Longitudinal cable or conductor tear at approx. $9.8 \mathrm{~m} / \mathrm{s}^{2}$ separation acceleration. 


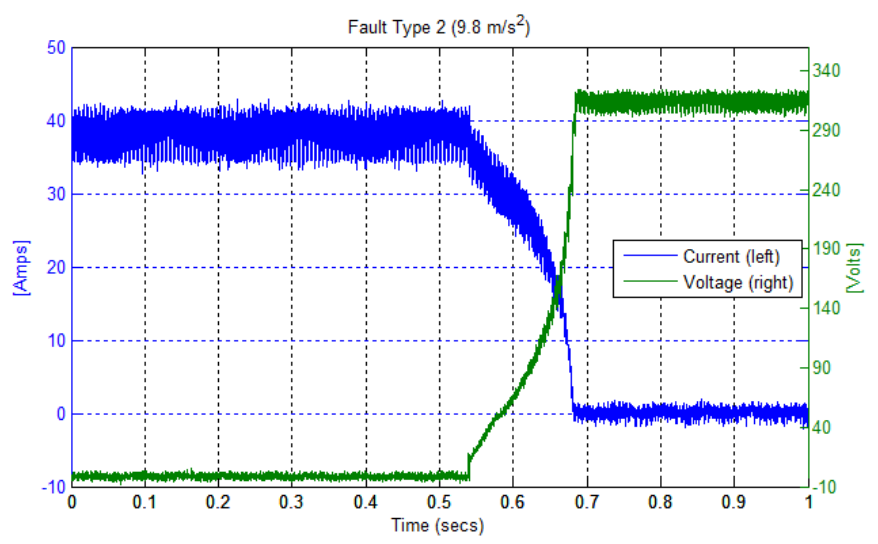

Figure 12. Experimental gap voltage and current for fault in Fig. 11.

\section{B. Planned Experiments}

The investigation described above is part of a whole series of planned experiments of growing complexity aimed at the full commissioning of the microgrid. The microgrid has been used to provide experimental validation of instabilities in dc distribution system with instantaneous constant power loads [1]. Illustrating the value of larger scale experimental validations, the microgrid tests failed to demonstrate the high frequency instabilities predicted by simulation and observed in small scale testing [2]. Other tests to determine the microgrid stability when loaded with a constant power load are also under way. Other planned research activities include

- Study of the transients induced by islanding of the microgrid and its subsequent reconnection to the power system

- Optimization of control methods for the active microgrid components for best power quality at the loads and least impact on the system at large

- Development of effective protection strategies for the microgrid and fault management solutions

- Integration of energy storage elements in the microgrid

- Interface with nontraditional energy resources

- Study of the microgrid behavior in the presence of pulsed power loads.

UT-CEM is engaged in a multi-phase approach to commission and demonstrate a microgrid based on either a dc bus or a high frequency ac power distribution network. This sequence started with a subscale low voltage low power setup intended to commission the controllers needed for full-scale medium voltage experiments. Each experimental setup will progressively increase in complexity and will be used to verify and correct system level simulation models. The completion of this planned sequence of experiments will commission the microgrid based on a dc power distribution architecture as shown in Fig. 1 or equivalently on a HFAC bus.

\section{CONCLUSIONS}

The ongoing development of a microgrid at UT-CEM has been described, current simulation and experimental work has been reported, and plans for future activities have been outlined. The microgrid will serve as a flexible test bed to study components and subsystems and their interactions at the MW power level, both within an MVDC and an HFAC architecture. This is of crucial importance to shipboard power systems especially as the concepts of the all-electric ships evolve, but are also of particular interest to land-based installations as well.

The assembly and sectional tests of the microgrid are continuing, accompanied by studies, simulations, and validation of the predictive tools developed. Ultimately, the intent is to use the UT-CEM microgrid as a flexible test bed that will shed light on issues like stability, reconfigurability, fault management, survivability, and the integration of renewables and energy storage that are critical to the implementation of a reliable, autonomous, and intelligent electric power system for a ship.

\section{ACKNOWLEDGEMENTS}

UT-CEM gratefully acknowledges the Office of Naval Research for their support of the ESRCD microgrid facility under grant N00014-08-1-0080. We would also like to acknowledge ONR for the two DURIP grants - N00014-08-10796 and N00014-080100798 - which enabled the Energy Storage Instrumentation System and High Speed Megawatt Dynamometer currently being used in support of the microgrid experimental program.

\section{REFERENCES}

[1] Alexis Kwasinski, Chimaobi N. Onwuchekwa, "Dynamic Behavior and Stabilization of dc Micro- grids with Instantaneous Constant-Power Loads," accepted for publication, IEEE Transactions of the Power Electronics Society.

[2] R. Hebner, J. Herbst, and A. Gattozzi, "Intelligent microgrid demonstrator," Electric Machines Technology Symposium 2010 (EMTS2010), Philadelphia, Pennsylvania, May 19-20, 2010. 